\title{
Research on Hill Start Control for Heavy Truck with AMT
}

\author{
Jiang Xuefeng ${ }^{1,2}, \mathrm{Xu} \mathrm{Xian}^{2}$, Jin Guodong ${ }^{1}$, Chen Wei ${ }^{1}$ \\ 1. Huazhong University of Science \&Technology, Wuhan, China \\ 2. Dongfeng Commercial Vehicle Technical Center, Dongfeng Motor Co., Ltd. \\ E-mail: chenw.hust@gmail.com
}

\begin{abstract}
The start control strategy is the key technology of the AMT, and the hill start control is the very difficult for the AMT control. This paper presents the research on hill start control for the heavy truck with the AMT. The model was built with Matlab/Simulink, and the simulation was carried out to study the performance of the vehicle. The result shows the effectiveness of the hill-start control strategy.
\end{abstract}

\section{Keywords-AMT, Hill Start, Modeling, Simulation}

\section{INTRODUCTION}

The AMT (Automatic Mechanical Transmission) has attracted increasing interesting from automotive researches, because it has some advantages of MT(Manual Transmission) and AT(Automatic Transmission), such as low cost, high efficiency, easy to use and good comfort. And the AMT can be manufactured by manual transmission product line with low equipment investment $[1,2]$. Thus, the heavy trucks equipped AMT are spreading in recent years, especially in Europe, Japan and China.

The hill-start is very important for the AMT. The vehicle will move backward, start with jerk, or cause engine stalling if failed on the slope road [3]. For the manual transmission, the hill-start depends on the driver's skilled driving technology to coordinate the brake pedal, the clutch pedal and the throttle pedal to achieve a smooth start. However, the AMT removes the clutch pedal, thus the driver can't perceived the clutch position. So it is difficult to hill-start for the driver with AMT without hill-start control strategy.

There have been some researches with hill-start control strategy. The Toyota use the HAS (Hill-start Assist System) to achieve the hill-start. It uses a sensor to monitor the wheel state. If the wheel begins to roll backwards, the ECU controls the brake the system to engage the brake on the wheel [4]. However, the hill-start is not perfect, because it can't avoid the rolling back. Ge [5] proposed an optimal control strategy by identification the driving resistance, however, the driving resistance is not easy to achieved when the vehicle drives on the road. Wang[6] designed a hill-start control strategy with the reconstruction of the breaking system. However, the strategy can't coordinate the clutch, brake and the engine very well. It will also cause vehicle move backwards or engine stalling. This paper presents a new control strategy for the heavy truck with the AMT. Compared with other types of hill-start strategy, the control strategy in this paper have some advantages such as more safe, start smoothly and less impact when start.

The paper is orgnized as follows. The section 2 shows the modeling of the drivelines including engine, clutch, gearbox, wheels, etc. The hill start control stragey was described in section 3. The controlled AMT model was simulated in section 4. The simulation results show the effectiveness of the control stragey. And section 6 gives the conclusion and further study.

\section{COMPONENT MODELS}

The vehicle equiped AMT system consists of complicated component subsystems such as the engine, clutch, gears, wheels and vehicle road load. These component models are described in the following.

\section{A. Engine model}

It is very diffcult to described a very detailed engine model. Because there are combustion, thermo dynamics, friction, etc. inside the engine. To simplify the modeling porcess, this paper use the experiment data to represent the engine [7]. The output torque of the engine is interpolated corresponding to engine speed and throttle position from an engine map modeled as a 2 dimension look-up table. For each throttle opening, the engine torque from idle to redline is a function of engine angular velocity, which is discribed in the following:

$$
T_{e}=f\left(\alpha, w_{e}\right)
$$

Where, $T_{e}$ is the output torque of the engine, $\alpha$ is the throttle posion which is from 0 to 1 , and $w_{e}$ is the rotational speed of the engine.

\section{B. Cluch model}

The function of an engaging friction clutch is to transmit torque gradually, to avoid high accelerations or jerks, when the engine is connected to the rest of the driveline. This torque is transferred from the engine through the pressure plate onto one or more friction plates connected to the transmission input shaft. There are three distinct modes of clutch: free, where the two plates transmit no torque; slipping, where the two plates have differing angular velocities; and lockup, where the two plates rotate together.

The torque capacity of the clutch is a function of its size, friction characteristics, and the normal force that is applied [8].

$$
T_{f \max }=\mu F_{n} n\left(\frac{2}{3}\right)\left(\frac{R_{0}^{3}-R_{i}^{3}}{R_{0}^{2}-R_{i}^{2}}\right)
$$

Where ${ }^{\mu}$ is coefficient of friction that can be formulated as a function of clutch slip, $F_{n}$ is normal force on clutch face that depends on the apply pressure, $R_{o}$ is outside radius of 
friction disc, $R_{i}$ is inside radius of friction disc, $n$ is the number of friction discs.

When the clutch is slipping, the clutch systems can be described as follows:

$$
\begin{aligned}
& J_{c} \dot{\omega}_{c}=T_{c}-T_{d}-b^{*}\left(\omega_{c}-\omega_{t}\right) \\
& T_{d}=\left(\omega_{c}-\omega_{t}\right)
\end{aligned}
$$

The torque through the clutch while slipping is given by:

$$
T_{c}=F_{\mathrm{n}} \mu R_{a} \operatorname{sign}\left(\omega_{\mathrm{e}}-\omega_{t}\right)
$$

In which $\mu$ is the friction coefficient of the clutch surface material, $R_{a}$ is the active radius of the clutch plates and the normal actuation force on the clutch plate is given by $F_{n}$.

When the clutch is sticking, the engine degree of freedom is rigidly coupled to the clutch disk at the friction interface. The clutch systems can be described as follows:

$$
\left(I_{e}+I_{v}\right) \dot{\omega}_{e}=T_{e}-T_{d}-b_{c}\left(\omega_{e}-\omega_{t}\right)
$$

The sticking of the clutch sustains as long as the torque transmitted through clutch remains below the maximally transmittable torque $T_{f \max }$.

\section{Gear model}

The gears provide speed and torque conversion from a rotating power source to another device using gear ratios. Considering the power loss caused by the friction and gear teeth contact, the gear model can be described as:

$$
\begin{aligned}
& T_{\text {out }}=\gamma T_{\text {in }} \\
& \omega_{\text {in }}=\gamma \omega_{\text {out }}
\end{aligned}
$$

Where, $T_{\text {in }}$ is the torque acted on the input shaft, $T_{\text {out }}$ is the output torque, $\omega_{\text {in }}$ and $\omega_{\text {out }}$ are the rotary speed of the input gear and output gear,respectively. $\gamma_{\text {is }}$ the transmission effectioncy.

\section{Wheel model}

The model structure we assume that the driving wheels and tyres are modeled as an inertia connected to the vehicle mass through a linear stiffness with coefficient $b$. This damper forms the slipping traction interface between tyres and road. A non-linear tyre traction model is proposed as a function of the tyre slip $S$ :

$$
s=\omega_{w}-\frac{v_{v}}{R_{w}}
$$

where $\omega_{w}$ is the wheel speed, $R_{w}$ is the dynamic wheel radius and $v_{v}$ is the vehicle speed.

\section{E. Vehicle system model}

The longitudinal vehicle road load includes the rolling resistance, aerodynamic resistance, and uphill resistance. The vehicle model can be described as follows:

$$
m a=F_{t}-F_{r}
$$

$$
F_{r}=\mu m g \cos (\alpha)+\frac{1}{2} C_{d} A v^{2}+m g \sin (\alpha)
$$

Where $F_{t}$ is the driving force on the vehicle, $F_{r}$ is the total resistance, $m$ is the mass of the vehicle, $a$ is the acceleration, $\alpha$ is the grade angle, $\mu_{\text {is the friction coeffient }}$ of the wheel, $C_{d}$ is the air drag coefficient that depends on the body style and dimension and $A$ is the frontal area of the vehicle.

\section{Hill Start CONTROL STRATEGY}

The hill start was achieved by the brake systems, as can be seen in Figure1. When the vehicle is prepared to start up on a slope road, the driver push the hill-start pushbutton to change the vehicle into hill-start mode. The control strategy changes the gear to a proper gear number by pneumatic actuator. When the driver release the hand brake, the air is prepared to pass through the abs valve. However, the abs valve is controlled by the VECU module, which controlled the abs valve by engine torque, gearbox, and the hill-start pushbutton. If the output torque of the engine doesn't achieved the threshold value, the abs valve won't open to let the air pass through to release the brake chamber. The brake system lock the wheel to roll back. So the vehicle remains on the slope road, seen in Figure2.

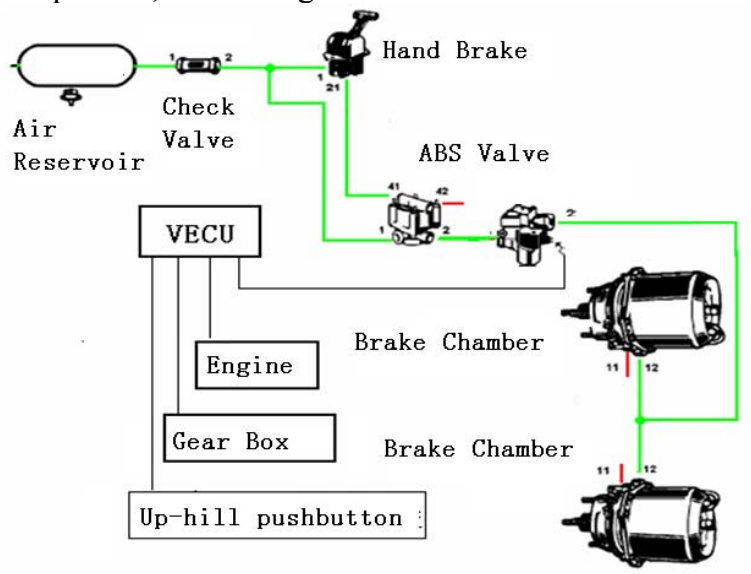

Figure 1. The hill-start system

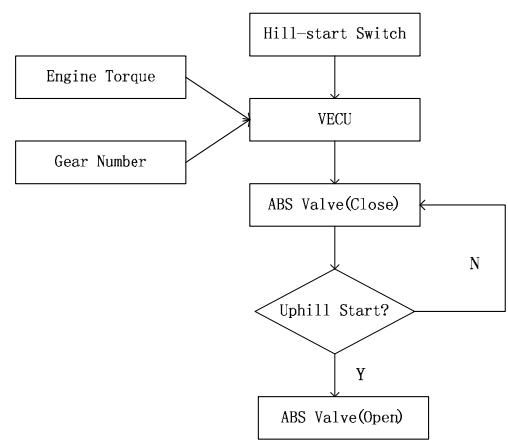

Figure 2. The control strategy 
When the driver press the throttle pedal, the output torque of the engine will increase according to the throttle position. When the engine torque is above the threshold value, the VECU controlled the ABS valve to open to let the high pressure gas pass throught, and release the brake chamber. So the vehicle will move on the road. The increase of the engine torque is proportional to the decrease of brake force.

The advantage of the control strategy is that, firstly, if the engine torque is not achieved the threshold, the vehicle will remain in the initial position, second, if the driver press the throttle pedal deeply, the engine torque will increase very fast, then the vehicle will start quickly.

\section{SimULATION AND RESUlts}

A complete heavy truck was built by Matlab/Simulink. The vehicle components model was assembled together to model a total vehicle, which can be seen in Figure 3. The vehicle was modeled by a forward-facing approach include the engine mode, clutch, gears, wheels and vehicle mentioned above. The heavy truck was equipped with a V8 engine with $1800 \mathrm{Nm}$ maximum torque and $310 \mathrm{~kW}$ power.

The vehicle with full load start on a road with $20 \%$ grade angel. The iput and output speed of the clutch can be seen in Figure4. It shows that the vehicle start up smoothly. The Figure 5 shows the impact of the vehicle. The impactness of the vehicle is $14.5 \mathrm{~m} / \mathrm{s}^{\wedge} 3$, and the less than 17.6 , which is defined by chinese standard. It shows that the vehicle can start up on the slope road very smooth, and with less impact.

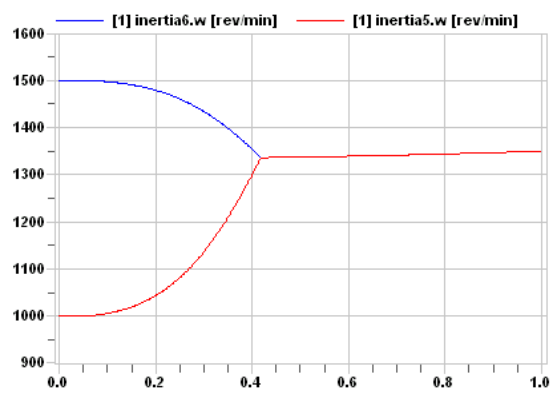

Figure 4. The input and output speed of clutch

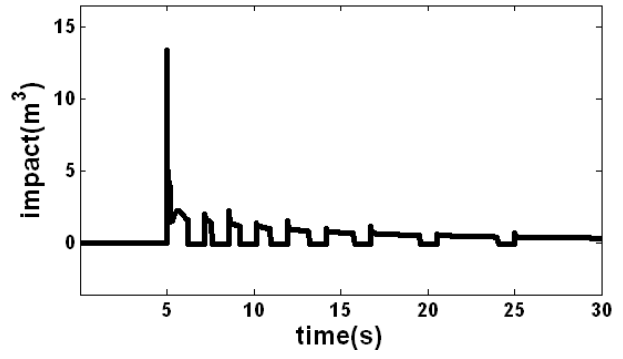

Figure 5. The impact of the vehicle

\section{COCLUSION AND FURTHER STUDY}

The hill start control is very important for the AMT control. This paper presents a control strategy for the heavy truck with the AMT. Compared with other types of hill-start strategy, the control strategy in this paper have some advantages such as more safe, start smoothly and less impact when start. The model was built with Matlab/Simulink, and the simulation was carried out to study the performance of the vehicle. The field test will carried out in the future on the vehicle to validate the effectness of the control strategy.

\section{ACKNOWLEDGEMENT}

This work was supported by the National High-Tech R\&D Program, China (No. 2009AA044501).

\section{REFERENCES}

[1] Hiroshi K., Naoyuki O. Takashi O. and Masaru Y. Next-generation Fuel-efficient Automated Manual Transmission. Hitachi Review, 2004, Vol. 53, No. 4.

[2] Gianluca L., Marcello M., Carlo R. Modelling of an automated manual transmission system. Mechatronics, 2007, 17: 73-91

[3] Zhang L., Li H. Hilling Start-up Control Method Using Accelerometer. Vehicular Electronics and Safety, IEEE International Conference, 2006, 276 - 279

[4] Taki Y., Yamada N.,Nagae A., Ishida Y. Development of Brake Control System for Driving Hills applying Active Wheel Speed Sensor. JSAE, 2003, Vol. 34 No. 3, pp.13-16

[5] Ge A., Lei Y., Gao Y., Zhang H. \& Fan J. A Research on Hill Starting Control of AMT System of Vehicle, Automotive Engineering, 1998, 3

[6] Wang H., Zhao X., Liu H. The electronic-pneumatic hill-starting assist control for heavy-duty vehicles with AMT. Journal of Shandong University (Engineering Science), 2009, 05

[7] Manish K., Taehyun S., Zhang Y. Shift dynamics and control of dualclutch transmissions. Mechanism and Machine Theory, 2007, Vol.42, pp.168-182

[8] Liu F, Li Y, Zhang J, Huang H. Robust Control for Automated Clutch of AMT Vehicle. SAE Paper 2002-01-0933 


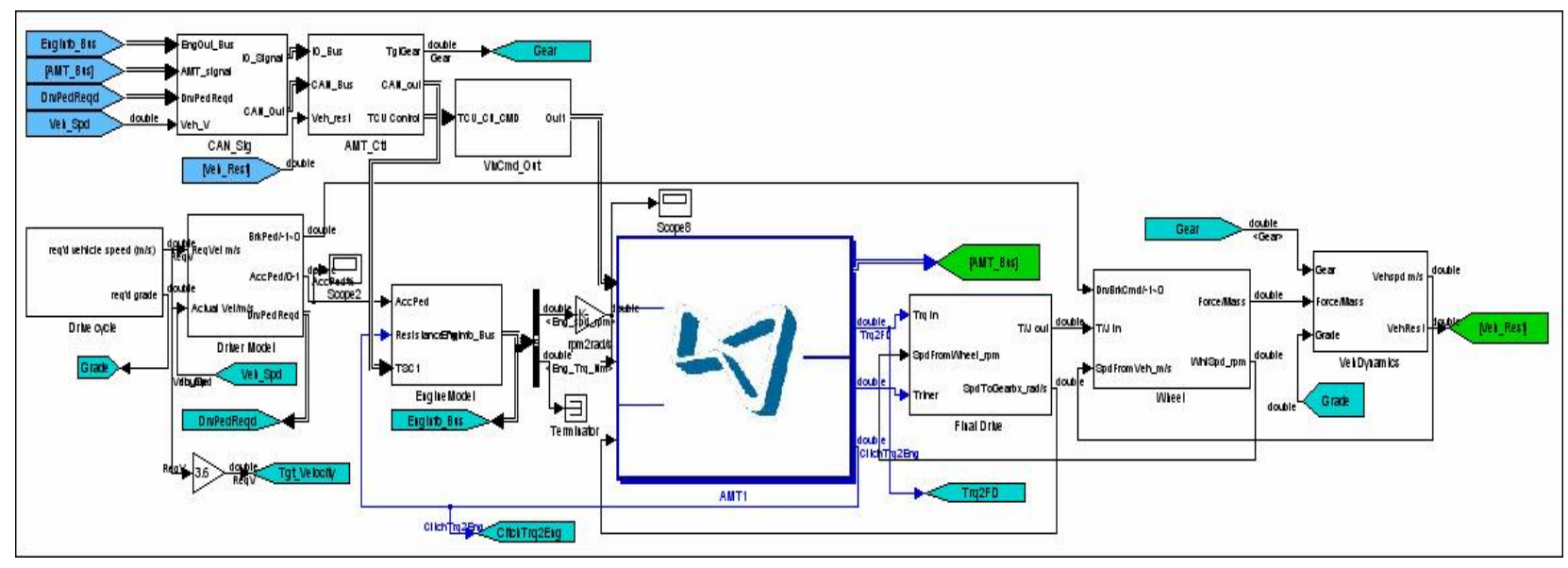

Figure 3. The vehicle model with Matlab/Simulink 